\title{
Measures of mortality in COVID-19: beyond the death toll
}

Prasad Chathuranga Weerasinghe ${ }^{1 *}$, Indumini Gunatilake ${ }^{1}$, Nirma Alpitiarachchi $^{1}$, Indika Gunawardana ${ }^{1}$, Ahmed Shiyam $^{1}$, Suwani Dasanayake', Gayani Dissanayake', Thanuja Ratnayaka ${ }^{1}$, Irshad Mashood, Disala Welgama', Jinadari Amarasena', Iresha Jayawickrama ${ }^{2}$, Charith Hettiarachchi', Ruwan Ferdinando $^{1}$, Mahendra Arnold ${ }^{1}$

${ }^{1}$ Ministry of Health, Sri Lanka; ${ }^{2}$ University of Lincoln, United Kingdom; ${ }^{3}$ University of Technology, Sydney, Australia

*Correspondence: prasad.chathuranga@gmail.com iDhttps://orcid.org/0000-0002-7499-0911

DOI: https://doi.org/10.4038/jccpsl.v26i5.8340

Received on 16 May 2020

Accepted on 25 May 2020

\section{Highlights}

- Different epidemiological measures of mortality are widely used to describe the effects of COVID-19 Pandemic globally in scientific and general literature. Each measure has their own limitations.

- Some measures like Case Fatality Ratio can give extreme values when the number of patients is low. Infection Fatality Ratio is an excellent alternative to identifying the disease's exact mortality since there could be undiagnosed cases that could be of relevance for the calculation.

- Dissemination and analysis of the mortality measures have to be done cautiously with other background information to avoid misinterpretations.

\section{Background}

The COVID-19 pandemic is currently identified as the biggest challenge that mankind has ever faced since World War II. Following its rise in Asia toward the end of last year, the infection has spread to each continent except Antarctica (1). As of 14 May 2020, there had been 4.5 million confirmed cases and 300,710 deaths reported globally; and new cases and deaths are continuously being reported from all around the world (2). Both confirmed cases and deaths are rapidly increasing in most of the countries showing an exponential growth (3-4). It is also reported that the true death toll has been higher than the number reported in many places since only a few countries are conducting adequate testing to identify all the coronavirus deaths. Deaths that occur outside a hospital can easily go undetected, untested, and undiagnosed. Even within a hospital setting, if a patient is not showing typical symptoms of severe COVID-19 illness, it can end up as an unreported death (5).

COVID-19 is different from other viral epidemics of 
the recent past in a few ways: it is more widespread than Severe Acute Respiratory Syndrome (SARS); more infectious than seasonal influenza; and has killed more people than Ebola. Number of deaths due to the pandemic is considered as an important measure since COVID-19 is widely spread all over the world compared to the SARS outbreak (5). The basic reproduction number $\left(\mathrm{R}_{0}\right)$, the average number of individuals contracting the infection from an infected person is higher (2.5) when compared to seasonal influenza (2.0) (6). Compared to Ebola, which killed 11,310 people between 2014 and 2016, COVID-19 is showing a much higher number of deaths $-287,399$ by 13 May 2020 (7). Further, it is different in the way that epidemiologists are tracking its progress. Instead of relying principally on the number of infections or the ratio of deaths to infections - known as the case fatality rate, researchers are looking at the daily deaths attributed to COVID-19 to monitor the impact of the disease and guide the response. Due to lack of testing in many countries and the virus's ability to spread in people who do not show symptoms, counting the number of infections has been very difficult. Therefore, since COVID-19 has also been more fatal than many other recent epidemics, understanding the pandemic mainly through its mortality parameters has been useful (8).

The simplest measure of mortality is the number of deaths. Since it is heavily influenced by the number of people who are at risk of dying, demographers typically measure mortality using rates. These rates are numerous; crude death rates, specific death rates, case fatality ratios, proportional mortality rates, survival rates and standardized or adjusted rates are some of them. 'Crude death rate' is the total number of deaths which occurred in a year, as a proportion of the total population of the same year (conventionally, the mid-year population). 'Age-specific death rate' is the number of deaths among a specific age group, expressed as a proportion of the total population of the same age group (9). Number of deaths occurring among individuals due to any specific condition (or a disease) given as a proportion to the total number who had that same condition, is known as the 'case fatality ratio'. The proportion of deaths attributable to a specific cause out of the total number of deaths that happened during the same period is known as the 'proportional death rate'. Standardized or ageadjusted death rates are useful for comparisons among populations with different age compositions (10). 'Survival rate' is a frequently used measure in conditions like cancers. It is the percentage of individuals living after a period of time (usually 5 years) in a treatment or follow-up group with the disease/condition (11). In addition to those, 'years of potential lives lost' (YLL) is used as a cumulative measurement of the life years lost due to premature deaths. In some instances, it is calculated per death, to identify the average life years lost due to premature deaths by an individual. However, in COVID-19, the total number of deaths and other related parameters going beyond the often-used repertoire of mortality measures seem to be of value in assessing the impact, progression and the severity of the disease. They may be useful tools to learn about the risk factors of diseases as well as to compare the measures taken across different populations. It is predicted that in the absence of interventions, COVID-19 would have resulted in 7.0 billion infections and 40 million deaths globally this year (3). The improvised mortality measures will shed light upon the epidemic and its control (12). In this backdrop, the objective of this article is to discuss the importance of mortality measures as indicators of the impact of the pandemic and interventions done.

\section{Different mortality measures used in COVID-19}

\section{Death count/total number of deaths per day}

The total number of deaths in a day has emerged as a useful statistic for tracking the progress of COVID19 in different regions. The rate at which the daily death counts increase or decrease is an indicator of what the virus is doing in a population (8). The trends in the cumulative deaths in a country give a clear indication as to whether the epidemic is under control or whether more stringent measures need to be adopted. The cumulative number of daily deaths due to COVID-19 is considered a more accurately reported measure over the daily number of confirmed cases. However, it starts to rise only after two weeks from the point of surge of cases. In such instances, 
early identification of exponential growth is easy when the logarithmic scale is used (13).

In addition, graphs can be used to observe when the bending of the curve occurs and are important to investigate the relative effectiveness of different interventions across countries (14). The cumulative death graphs also indicate the current and past trends and whether they are about to get flattened in relation to deaths. In Figure 1, it is clearly visible that daily deaths are still rising in the USA while in other countries, there is a decline or flattening trend as of 8 April 2020 (8).

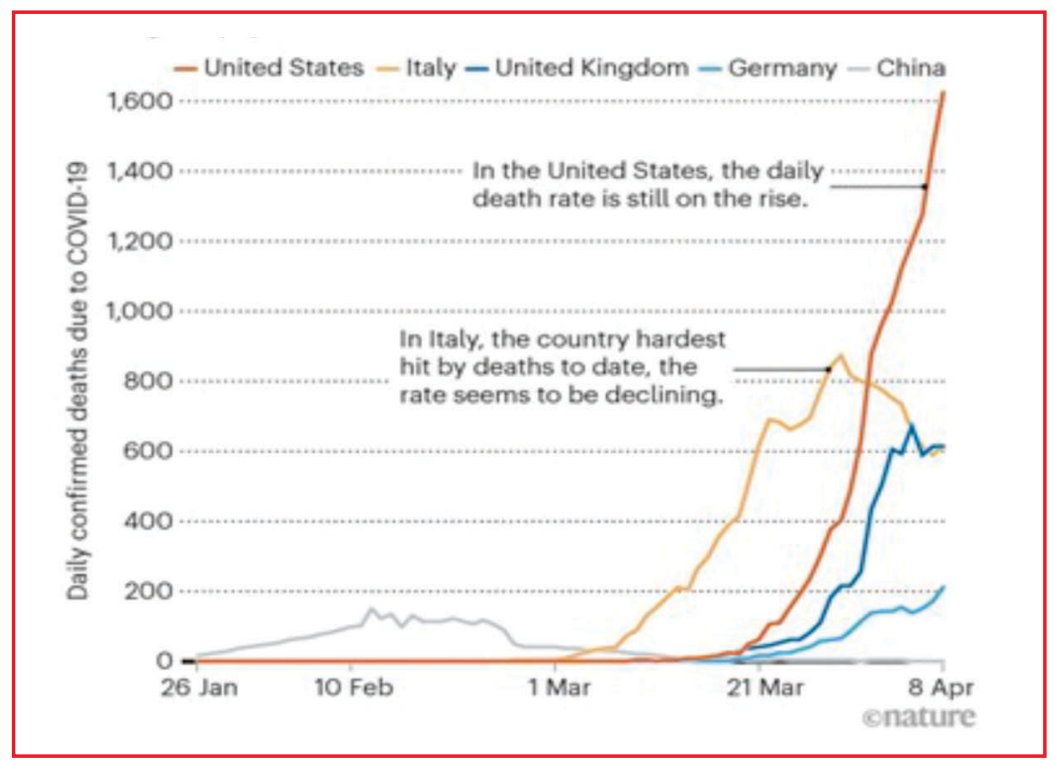

Figure 1: Mortality trend in selected countries (8)

The case definition is of paramount importance in comparing these numbers and trends across the countries. These case definitions have been updated several times with changing disease patterns from the beginning of the epidemic in China. There are differences in the case definitions among countries according to the local stages of the spread. Following the new guidelines of Centres for Disease Control and Prevention (CDC), as of 14 April 2020, CDC case counts and death counts include both the confirmed and probable cases and deaths (15). According to the latest Sri Lankan guidelines, six scenarios have been agreed upon to classify one to be suspected as COVID-19, with different clinical presentations and contact/travel history combinations. Suspected cases are investigated, and confirmation of the diagnosis is done through polymerase chain reaction (PCR) testing. Labelling as a confirmed case is done solely based on the PCR result, irrespective of the clinical history (16).

\section{Case fatality ratio (CFR)}

This is a frequently used measure to assess the fatality of a communicable disease. In epidemiological terms, it is defined as the proportion of cases of a specified condition that are fatal within a specified time; also called the 'case fatality ratio' (CFR) (17). It can be calculated as a proportion of those who died due to the disease among those who acquired it (10). Simply, it can be expressed by the following equation.

Case fatality ratio $=$

No. of deaths due to the disease $* 100$

No. of diagnosed patients

Calculation of the true case fatality ratio of COVID19 , by applying the currently diagnosed number of patients and deaths reported as COVID-19 to the above formula is not practical. Current outcomes are related to past cases due to the delay in the 
progression of the disease. Further, the accuracy of data and the proportion of population tested are different in various regions, and therefore the comparison is challenging. The higher CFR value reported in Wuhan, China may be a result of an overestimate caused by the under-reporting of milder and asymptomatic cases. Delays in the testing of patients having symptoms of the novel corona viral infection and being in the early stage of the epidemic (where the deaths are yet to occur), can cause an underestimation of the CFR in other regions (18).

The CFR is not constant, and it changes with the context. It reflects the severity of the disease in a particular context, at a particular time period and in a specific population; and does not depend only on the disease but also on the quality of treatment received and the capacity of the health system to recover from it. The CFR can decrease or increase over time as responses change; and it can vary by location and the characteristics of the infected population, such as age or sex and those with pre-existing diseases. Therefore, the elderly would expect to have a higher CFR from COVID-19 than the younger age groups.

To calculate the final CFR according to the above formula, the outcome of each patient (recovered or dead) needs to be known. In COVID-19, there are $58.08 \%(\mathrm{n}=2,263,086)$ among the total diagnosed cases who are still alive up to 7 May 2020 and their outcome remains uncertain (3). If it is calculated using the currently diagnosed number of patients and the current number of deaths, it is assumed that all the patients who are still having the disease are going to recover. According to the above assumption, the global case fatality ratio would be $6.91 \%$.

\section{Confirmed deaths relative to the size of the population (crude death rate)}

It can be insightful to know how many people have died compared to the total population living in that country. For instance, if 1000 people died in Iceland out of a population of about 340,000 , that would have a far greater impact than the same number dying in the USA, with its population of 331 million. Therefore, the death counts in more populous countries tend to be higher (19).
When calculating the mortality rate, we need to consider the number of actual cases (not merely the reported ones, which will typically represent only a small portion of the actual ones) that have already had an outcome (positive or negative: recovery or death), and the number of actual deaths related to the closed cases examined. As of 1 May2020, 23,430 people are estimated to have died out of a total population of $8,398,748$ in New York City. This corresponds to a $0.28 \%$ crude mortality rate or 279 deaths per 100,000 population or 1 death in every 358 people. It is noteworthy that the crude mortality rate will continue to increase as more infections and deaths occur (3).

\section{The 'excess mortality'}

The World Health Organization (WHO) defines 'excess mortality' as mortality above what is expected based on the non-crisis mortality rate in the population of interest (20). It is therefore usually attributable to a crisis condition and expressed as a rate (the difference between observed and non-crisis mortality rates) or as a total number of excess deaths. This has become an important parameter in the COVID-19 pandemic since there can be a significant number of unreported deaths due to multiple reasons. Reporting only hospital deaths, missing deaths of untested COVID-19 infected individuals, false negative testing, getting the infection after a negative testing, poorly established and less accurate death reporting systems are some of the key issues to be concerned with when evaluating the impact of pandemic on the mortality rates. There can be an indirect increase of deaths too owing to its burden on the health care system and less attendance for health care. However, this pandemic situation can cause an indirect reduction of the mortality owing to lock downs and restrictions, deaths due to road traffic accidents and other risky behaviours, which could affect the measurement of 'excess deaths' unless adjusted (20-21).

Excess 1-year mortality associated with the COVID19 pandemic has been estimated related to different infection rates and various suppression levels of the spread in a population-based cohort study conducted in England. Number of excess deaths per 1-year duration were 2, 4 and 7 with related relative risks 
(RR) of 1.5, 2.0 and 3.0, respectively, when full suppression is present. On the other hand, without any interventions, the estimations were 146,996, 293,991 and 587,982, respectively for the RR of 1.5 , 2.0 and 3.0. This indicates the importance of continuous interventions to prevent the spread of the disease mainly among the high-risk age groups and people with underlying high-risk conditions (21).

\section{Specific death rates related to COVID-19}

\subsection{Death rates and ethnicity}

According to the latest figures reported from the
United Kingdom, there is an over representation of Black, Asian and minority ethnic groups (BAME) among the critically ill patients. Nearly one third (34\%) out of 5000 critically ill individuals belong to the BAME group, although their population is only $14 \%$ in the respective region. According to data released by the CDC on 18 April 2020,30\% of all the cases in the USA are among the Black Americans which can be considered as an over representation since they contribute to only $13 \%$ of the total population. Health inequalities are considered as a possible reason for this ethnic difference other than the genetic backgrounds (22).

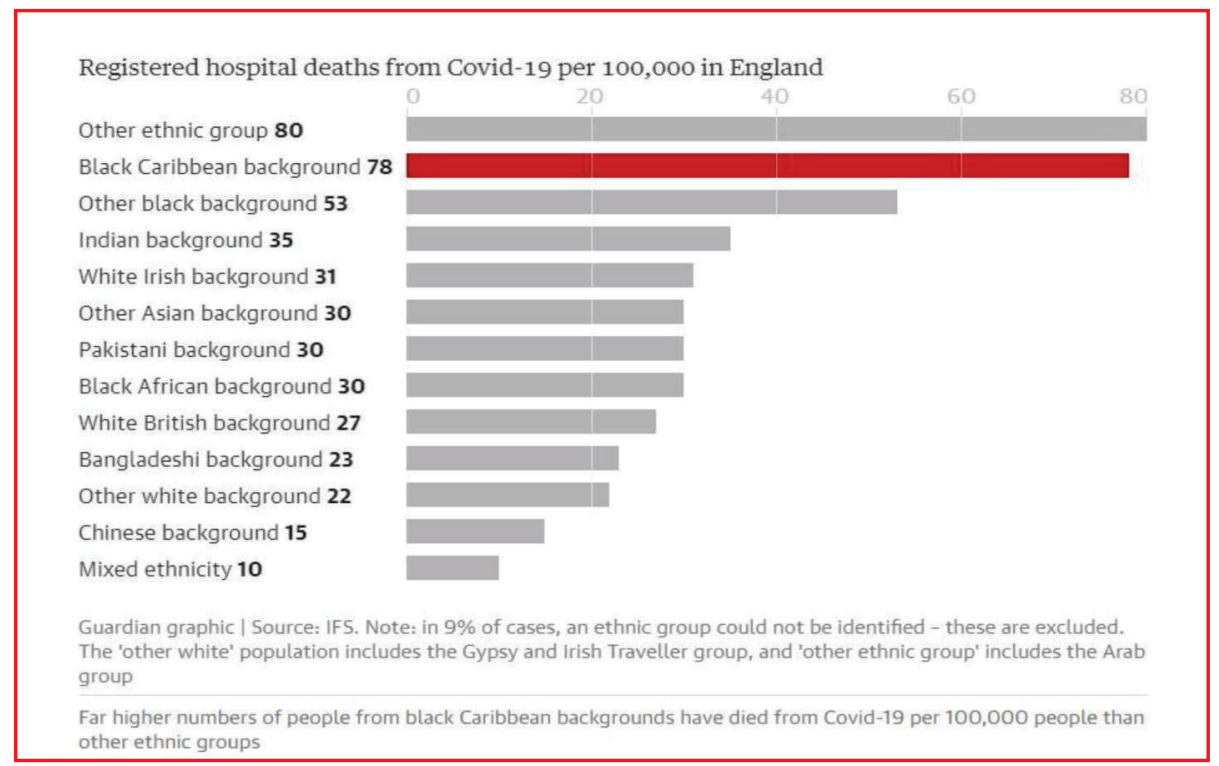

Figure 2: Mortality comparison among various ethnic groups in England (23)

Reasons for overrepresentation of the BAME people in COVID-19-related mortality and morbidity statistics can differ among the subgroups (Figure 2). These include differences in age structure, occupational exposures and different levels of economic vulnerability. Therefore, it is not possible to attribute these differences to a single causal factor (23).

\subsection{The case fatality ratio of COVID-19 by location}

The CFRs of COVID-19 vary widely across countries. However, it is not necessarily an accurate comparison of the true likelihood that someone with COVID-19 will die of the disease. According to the Report of the WHO-China Joint Mission on Coronavirus Disease 2019, the CFR has changed during the early period of the outbreak. In the earliest stages of the outbreak, the CFR was much higher. Seventeen per cent across China as a whole and greater than $20 \%$ in the epicentre of the outbreak in Wuhan, and in the weeks that followed, the CFR declined reaching as low as $0.7 \%$ for patients who first showed symptoms after 1 February 20202. CFR was different at different locations. By 1 February, the CFR in Wuhan was still $5.8 \%$ while it was $0.7 \%$ across the rest of China (24). 
In comparison, CFR in Sri Lanka was $0.88 \%$ with the reporting of the first COVID 19 death and a total of 113 confirmed cases in late March. It reached a peak of $3.7 \%$ with 189 total confirmed cases in around 10 days and nearly after one and a half months, the CFR has declined to $1.08 \%$ with 835 confirmed cases (Figure 3).

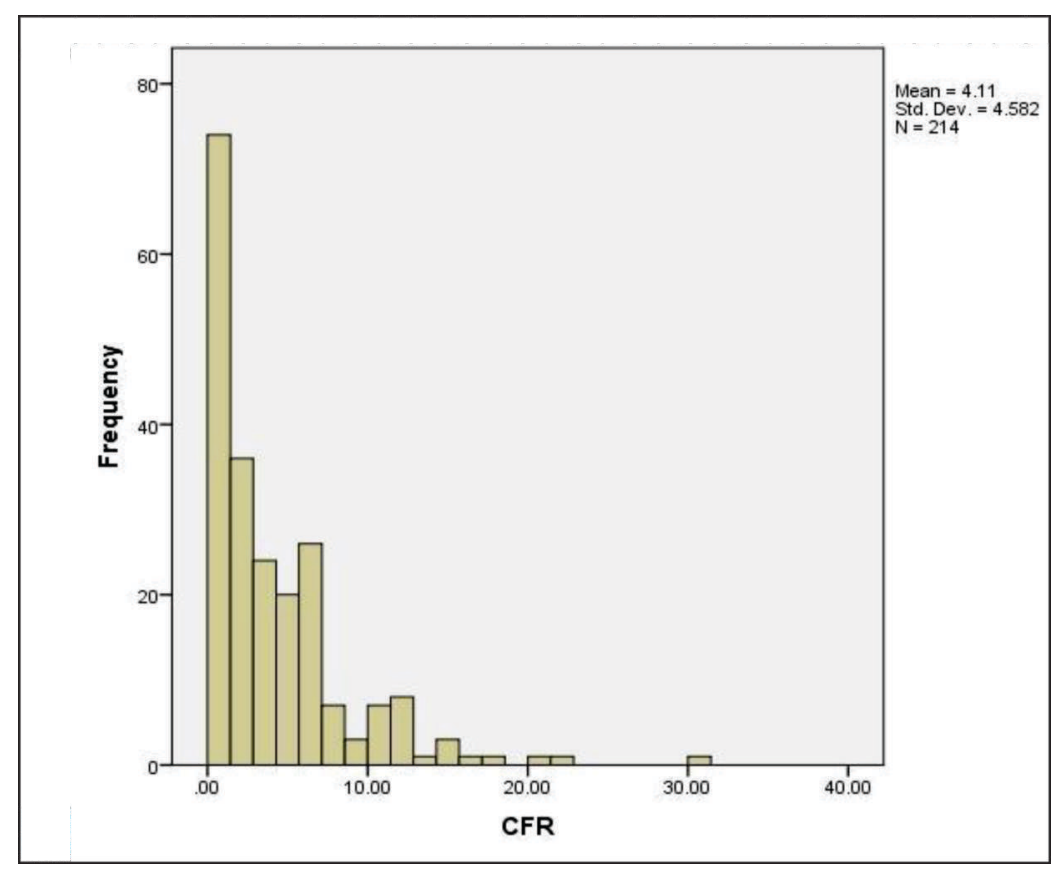

Figure 3: Country-wise distribution of the Case Fatality Ratios COVID-19 (25)

Distribution of the CFRs at country level is positively skewed with a mean of 4.1 and a median of 2.73 , with a huge variation. The country-specific CFRs for COVID-19 range from $0 \%$ to $31.25 \%$. This can be misleading due to extreme CFR values with a lower number of diagnosed patients. For example, CFR is $12.5 \%$ in the Mauritania because Mauritania has 1 death per 8 cases, while the USA has $5.92 \%$ $(n=74,809)$ of deaths among diagnosed individuals, with the highest number of cases $(1,263,243)$ reported globally up to 7 March 2020. The CFR for COVID-19 in Sri Lanka was $1.09 \%$ on 7 May 2020 (26).

\subsection{The CFR of COVID-19 by pre-existing health conditions}

The CFR for those with an underlying health condition is much higher than for those without. More than 10\% of those diagnosed with COVID-19 who were also having a cardiovascular disease died as result of the virus. Diabetes, chronic respiratory diseases, hypertension and cancer are the other common morbidities associated with increased fatality among COVID-19 patients (Figure 4). The CFR was $0.9 \%$ up to 11 February 2020 for those without any pre-existing health condition (4). The elderly are at higher risk of dying from COVID-19. This might be partly explained by the fact that they are more likely to have underlying health conditions such as cardiovascular disease, respiratory disease and diabetes: these health conditions make it more difficult to recover from the COVID-19 infection. 


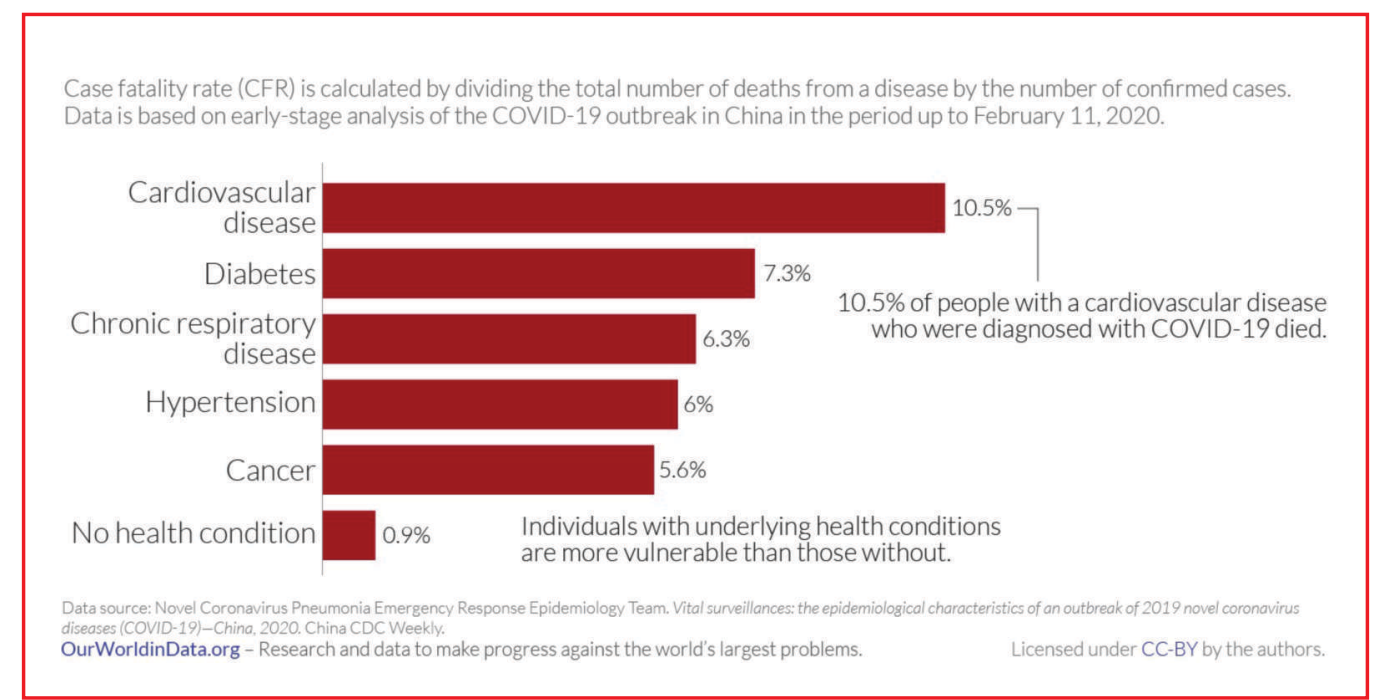

Figure 4: Coronavirus: early-stage case fatality ratios by underlying health conditions in China (27)

\subsection{Case fatality ratio of COVID-19 by age}

The CFR for total population does not indicate which age group within a population is most at risk. However, this understanding is crucial in an outbreak. Understanding the relative risk to different age categories in a population allows focusing on the most vulnerable population and improving the allocation of health resources to those who need them the most. Current data across countries suggest that the elderly are at a higher risk. Further, the CFR for people with underlying health conditions is shown to be higher than that for those without. One possible reason for this could be that elderly are more likely to have underlying health conditions.

\subsection{Case fatality ratio of COVID 19 compared to other diseases}

The CFR of MERS-CoV of 34\% by end of November 2019 (globally) is much higher, while the US seasonal flu with a CFR of approximately $0.1 \%$ is much lower than the current CFR for COVID-19 (28).

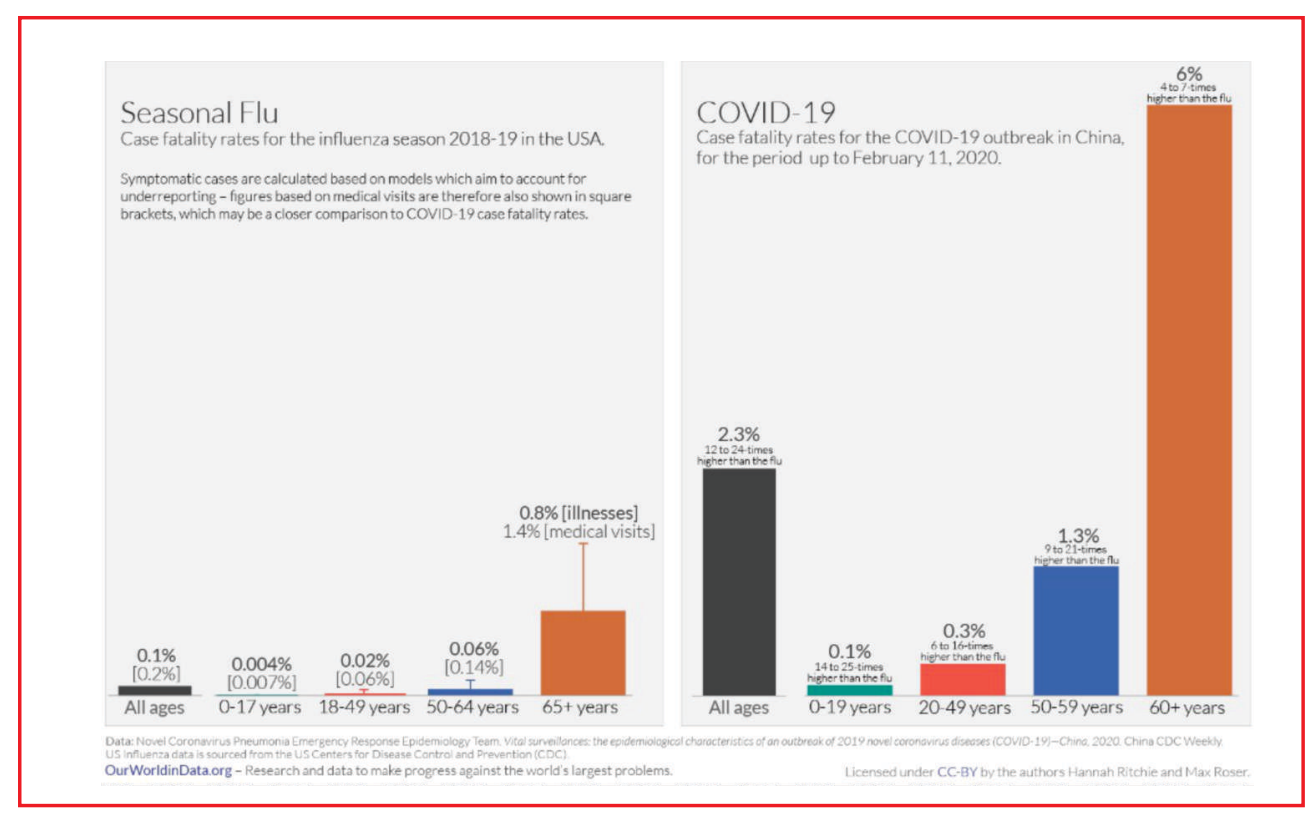

Figure 5: Case fatality ratios: COVID-19 versus US Seasonal Flu (19) 
A comparison has been done between the CFR during the outbreak of COVID-19 in China in 2019-2020 with CFR of the US seasonal flu in 2018-19 (Figure 5). The CFR of seasonal flu in the US is around $0.1 \%$ to $0.2 \%$, while the CFR for COVID-19 measured in the study was $2.3 \%$. However, as this is not the case for COVID-19 figures, it may be an unfair comparison. Mild cases may have been missed in the COVID-19 outbreak. However, this is likely to skew the comparison slightly in the other direction.

CFR for COVID-19 is much higher than the CFR of seasonal flu. The two diseases are similar in the profile of the fatality ratio by age where the elderly populations have a higher CFR. However, the CFR of COVID-19 is much higher for all age groups, including young people.

\subsection{Focusing on closed cases}

To avoid uncertainty of the outcome of the currently active cases, some use fatality ratios among the closed cases (cases with an outcome) as a descriptive measure of COVID-19

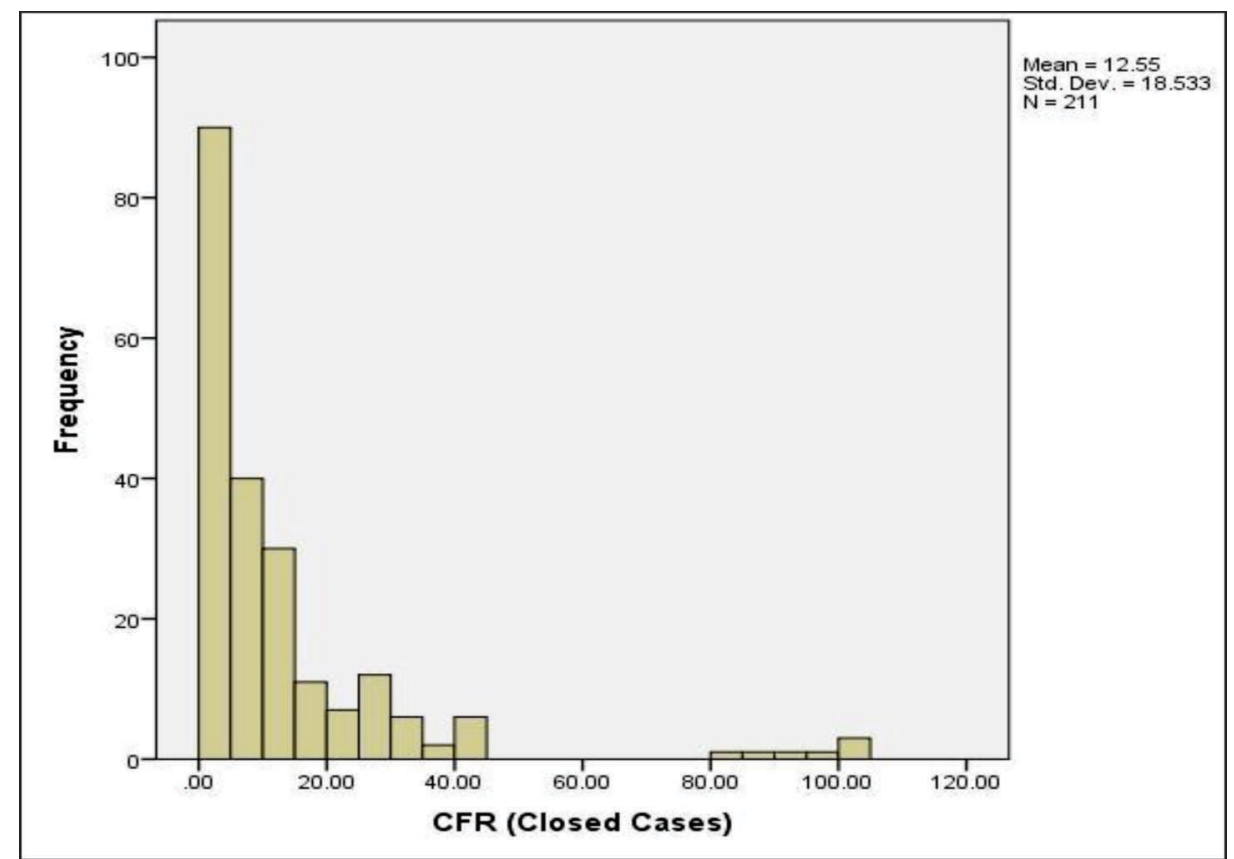

Figure 6: Country-wise distribution of the fatality ratios among closed cases of COVID-19 (25)

If it is to calculate the fatality among closed cases at global level, the average is $11.74 \%$ on 7 May 2020. When the fatalities among closed cases are separately analyzed for countries (with at least one closed case), the country-specific values show a wide variation. It ranges from 0 to 100 (mean 12.55 and median 6.71). Therefore, the global mean value of fatality among closed cases may not be applicable in local contexts.

Even if the value is given for a specific country, there can be extremely lower values with lower recovered cases. As an example, USA with the highest number of deaths $(74,809)$ has a fatality ratio of $25.9 \%$ among closed cases, while the Comoros show $100 \%$ fatality ratio with one death as of 7 May 2020 (25).

\section{Infection fatality ratio (IFR): a way to tackle the undiagnosed}

Parameters like CFR, fatality among closed cases and the recovered proportion among closed cases depend on the number of diagnosed patients (cases). According to the $\mathrm{WHO}_{2} 80 \%$ of infected individuals have a milder form of the disease and some can be asymptomatic (29). The number of diagnosed cases depends on the surveillance case definition as well as the availability and usage of the diagnostic facilities. Total infected individuals in the community with COVID-19 can be calculated by using mathematical methods (models) (30). These methods are designed based on the current pieces of evidence on disease 
transmission patterns. After calculating the total number of individuals with the infection, the following equation can be used to calculate the infection fatality ratio (IFR); the proportion of deaths due to COVID-19 among individuals who got the infection.

Infection fatality ratio $=$

No. of deaths occurred due to the disease $* 100$

Total no.of infected individuals

The current estimate of the IFR for COVID-19 ranges from $0.3 \%$ to $1 \%$ as at 19 February according to the WHO (30). These estimates depend on the accuracy of the mathematical model used. It can vary with the accuracy of the available data on disease transmission patterns used to build the model. The only available method for finding the exact number of individuals with the infection is testing at population level (population-level serological studies). IFR can be used to overcome the challenge of comparison among settings with different testing strategies and capacities (31).

\section{Death rate among patients admitted to hospital (hospital fatality rate)}

Death rates also can be calculated using the number of hospital admissions as the denominator. This is usually expressed with the rates of ICU admissions. This is not a measure for the general COVID-19 mortality since this has a selection bias (27). According to the news reported on 29 April 2020, $33 \%$ had died among a sample of 17,000 patients admitted to UK hospitals and $49 \%$ discharged while the rest was still getting treatment (32). Case fatality among the hospital admissions remains in between $35 \%$ to $40 \%$, which is equal to fatality rates of Ebola among hospital admissions (33) .

\section{Applicability of the evidence to Sri Lanka}

In a setting with a comparatively lower number of tests done proportionate to the population like Sri Lanka, mortality parameters can shed light on the progression of the epidemic as well as the effectiveness of the interventions made, if the missing or undiagnosed cases remain constant. Also, in an event of community transmission leading to a higher number of deaths, these mortality measures are important to identify the differentials between different groups. Dissemination of more informative mortality data such as age-specific death rates, ethnicity-specific death rates along with background information like the total number diagnosed is helpful to better understand and compare the scenario with other regions.

\section{Conclusions}

Epidemiological measures of fatality are used widely in assessing the impact of the disease as well as its response to the COVID-19 pandemic. Usage of CFR or fatality among closed cases to describe the current on-going pandemic of COVID-19 must be done with caution. Since there is a wide variation among countries, the use of global means alone may not be suitable. Country-wise calculations can give extreme values in the lower number for diagnosed or closed cases. Provision of additional information can be helpful to prevent misinterpretations. Accuracy of the IFR including the estimated numbers of infected individuals based on mathematical models can be tested only by population-based serological studies.

\section{Author Declaration}

\section{Acknowledgements: None}

Author contributions: All authors were involved in planning of the article and in literature searches. WMPCW drafted the manuscript and all authors involved in editing it. SMA and RF did the overall supervision. All authors went through the final manuscript.

\section{References}

1. UNDP. Coronavirus disease COVID-19 pandemic. UNDP 2020. Available from: https://www. undp.org/content/undp/en/home/coronavirus.html. Accessed 9 May 2020.

2. World Health Organization. WHO Coronavirus Disease (COVID-19) Dashboard 2020. Available from: https://covid19.who.int/. Accessed 9 May 2020. 
3. Worldometer. Coronavirus Cases-Worldometer 2020. Available from: https:// www.worldo meters.info/coronavirus/coronavirus-cases/\#dailycases. Accessed 7 May 2020.

4. Our World in Data. Linear vs. exponential growth: how do they compare?. Available from: https:// ourworldindata .org/ grapher/ linear-vsexponential-growth? time $=1 . .5$. Accessed 8 May 2020.

5. Kamel Boulos MN, Geraghty EM. Geographical tracking and mapping of coronavirus disease COVID-19/severe acute respiratory syndrome coronavirus 2 (SARS-CoV-2) epidemic and associated events around the world: How $21^{\text {st }}$ century GIS technologies are supporting the global fight against outbreak. International Journal of Health Geographics 2020; 19(1): 1-12. DOI: 10.1186/s12942-020-00202-8.

6. WHO. Q\&A: Influenza and COVID-19- similarities and differences. Available from: https://www.who. int/emergencies/diseases/novel-coronavirus2019/question-and-answers-hub/q-a-detail/q-asimilarities-and-differences-covid-19-andinfluenza? gclid= Cj0KCQjw-_j1BRDkA RIs A J c fm TF 2no4D 8 CmnitpMDWOx DDejNZqM4BGLsDb9vFxatE2JTPqU8rOGL4Ma Ai2zEALw_wcB. Accessed 15 May 2020.

7. United Nations. Learning from the past: UN draws lessons from Ebola, other crises to fight COVID-19. United Nations 2020. Available from: https:// www.un.org/ en/coronavirus /learning-past-undraws-lessons-ebola-other-crises-fight-covid-19. Accessed 8 May 2020.

8. Subbaraman N. Why daily death tolls have become unusually important in understanding the coronavirus pandemic. Nature 9 April 2020. DOI: 10.1038/d41586-020-01008-.1

9. Andrew H. The measurement of mortality. In: demographic methods. 1st ed. Arnold; 1998.

10. Centers for Disease Control and Prevention. Principles of epidemiology. Lesson 3 - Section 3. Available from: https:// www. cdc.gov/ csels/ dsepd/ss1978/lesson3/section3.html. Accessed 7 May 2020.

11. National Cancer Institute. Definition of survival rate - NCI Dictionary of Cancer Terms - National Cancer Institute 2020. Available from: https:// www.cancer .gov/ publications /dictionaries/c ancerterms/def/survival-rate. Accessed 15 May 2020.

12. Gt Walker $\mathrm{P}$, Whittaker $\mathrm{C}$, Watson $\mathrm{O}$, Baguelin $\mathrm{M}$, Ainslie KEC, Bhatia S, et al. The global impact of COVID-19 and strategies for mitigation and suppression 2020. Available from: https:/ /doi.org/10.25561/77735. Accessed 15 May 2020.

13. Johns Hopkins Coronavirus Resource Center. Cumulative Cases - Johns Hopkins Coronavirus Resource Center 2020. Available from: https://coronavirus.jhu.edu /data/ cumulative-cases. Accessed 16 May 2020.

14. Sam W, Adam V, Conrad Q, Harper LL. COVID-19 news: UK infection rate has risen in past week. New Scientist, 8 April 2020. Available from: https://www.newscientist. com/article/2237475covid-19-news-uk-infection-rate-has-risen-in-pastweek/.

15. Worldometer. Changes in US Data following new CDC guidelines on "case" and "death" definition. Worldmeter, April 14, 2020. Available from: https://www.worldometers. info/ coronavirus/usdata/. Accessed 16 May 2020.

16. Ministry of Health. GP Guideline Covid 19 Outbreak. Version 3.1. Available from: https:// drive.google.com/file/d/1KP43hywzGkr90ToNtga EcZaimuraH6uB/view. Accessed 16 May 2020.

17. Porta MA. Dictionary of Epidemiology. Oxford 2008.

18. Battegay M, Kuehl R, Tschudin-Sutter S, Hirsch HH, Widmer AF, Neher RA. 2019-novel Coronavirus (2019-nCoV): estimating the case fatality rate - a word of caution. Vol. 150, Swiss Medical Weekly 2020; 20203.

19. Our World in Data. Coronavirus (COVID-19) testing - statistics and research - our world in data 2020. Available from: https:// ourworldindata.org /coronavirus-testing. Accessed 13 May 2020.

20. Hannah R, Max Roser EO-O and JH. Excess mortality from the Coronavirus pandemic (COVID19). Our World in Data 2020. Available from: https://ourworldindata .org/excess-mortality-covid. Accessed 16 May 2020.

21. Olson DR, Huynh M, Fine A, Baumgartner J, Castro A, Chan HT, et al. Preliminary Estimate of Excess Mortality During the COVID-19 Outbreak - New 
York City, 11 March-2 May 2020. MMWR Morb Mortal Wkly Rep 2020; 69(19): 603-5. Available from: http:// www.cdc.gov/mmwr/ volumes/ 69/wr/mm6919e5.htm?s_cid=mm6919e5_w.

22. Liverpool L. An unequal society means COVID-19 is hitting ethnic minorities harder. New Scientist, 21 April 2020. Available from: https://www. newscientist.com/article/2241278-an-unequalsociety-means-covid-19-is-hitting-ethnicminorities-harder/.

23. The Guardian. British BAME COVID-19 death rate "more than twice that of whites". The Guardian 2020. Available from: https:// www. theguardian.com/world/2020/may/01/british-bamecovid-19-death-rate-more-than-twice-that-ofwhites. Accessed 16 May 2020.

24. World Health Organization. Report of the WHOChina Joint Mission on Coronavirus Disease 2019. Available from: https:// www. who.int/docs/defaultsource/ corona viruse/who-china-joint-mission-oncovid-19-final-report.pdf. Accessed 8 May 2020.

25. Center for Systems Science and Engineering (CSSE) at Johns Hopkins University (JHU). COVID-19 Dashboard. Available from: https://www.arcgis. com/apps/opsdashboard/index.html\#/bda7594740f d40299423467b48e9ecf6. Accessed 7 May 2020.

26. Health Promotion Bureau. Coronavirus (COVID19) Sri Lanka Analytics Dashboard 2020. Available from: https://hpb.health .gov.lk/c ovid19dashboard/. Accessed 9 May 2020.

27. Worldometer. Coronavirus death rate (COVID-19). Available from: https://www. worldometers. info/coronavirus/coronavirus-death-rate/\#hfr. Accessed 16 May 2020.

28. Guan WJ, Ni ZY, Hu Y, Liang WH, Ou CQ, He JX, et al. Clinical Characteristics of Coronavirus Disease 2019 in China. New England Journal of Medicine. 2020; 18: 1708-28. DOI:10.1056/ NEJMoa2002032.

29. World Health Organization. Q\&A on coronaviruses (COVID-19) 2020. Available from: https://www. who.int/emergencies /diseases/novel-coronavirus2019/question-and-answers-hub/q-a-detail/q-acorona viruses. Accessed 7 May 2020.

30. World Health Organization. Situation Report-30, 19 February 2020. Available from: https:// www.who.int/hac/crises/international/asia_tsunami /sitrep/30/en. Accessed 7 May 2020.

31. Henriques M. Coronavirus: why death and mortality rates differ. BBC, 2 April 2020. Available from: https://www.bbc.com /future/ article/20200401coronavirus-why-death-and-mortality-rates-differ. Accessed 16 May 2020.

32. The Guardian. Third of UK Covid-19 patients taken to hospital die, study finds. UK news, 29 April 2020. Available from: https://www. theguardian.com/uknews/2020/apr/29/study-finds-a-third-of-uk-covid19-patients-taken-to-hospital-are-dying. Accessed 16 May 2020.

33. Gallagher J. Coronavirus "as deadly as Ebola in hospital”. BBC News, 29 April 2020. Available from: https://www.bbc.com /news/health52473524. Accessed 16 May 2020. 